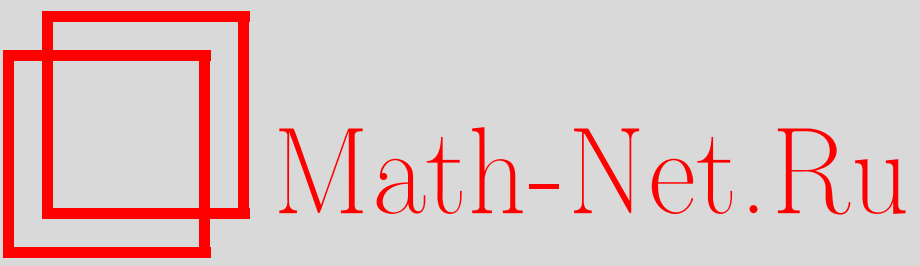

А. В. Цыганов, Неканонические преобразования времени, связывающие конечномерные интегрируемые системы, ТМФ, 1999, том 120, номер 1, 27-53

DOI: https://doi.org/10.4213/tmf758

Использование Общероссийского математического портала Math-Net.Ru подразумевает, что вы прочитали и согласны с пользовательским соглашением

http://www.mathnet.ru/rus/agreement

Параметры загрузки:

IP : 54.196 .121 .252

26 апреля 2023 г., 14:32:18 
ТЕОРЕТИЧЕСКАЯ

И МАТЕМАТИЧЕСКАЯ

ФИЗИКА

Том 120, № 1

июль, 1999

(C) 1999 г.

\section{НЕКАНОНИЧЕСКИЕ ПРЕОБРАЗОВАНИЯ ВРЕМЕНИ, СВЯЗЫВАЮШИЕ КОНЕЧНОМЕРНЫЕ ИНТЕГРИРУЕМЫЕ СИСТЕМЫ}

Рассматриваются дуальные штеккелевские системы, связанные друг с другом неканоническим преобразованием переменной времени. Доказано, что данная дуальность различных интегрируемых систем порождается неоднозначностью отображения Абеля. Построены матрицы Лакса и $r$-матричные алгебры для некоторых интегрируемых систем на плоскости. Подробно рассмотрены интегрируемые деформации задачи Кеплера и системы типа Холта.

\section{1. ВВЕДЕНИЕ}

В 80-х годах прошлого века в работах Ковалевской [1] были введены и изучены новые условия интегрируемости уравнений движения в квадратурах. Этот критерий позволил ей открыть третий общий случай полной интегрируемости уравнений движения тяжелого твердого тела с закрепленной точкой и ввести переменные разделения $\left\{q_{1}, q_{2}\right\}$. По-видимому, это было одним из первых примеров применения к конкретной физической задаче сингулярного анализа дифференциальных уравнений [2], который связывает глобальное свойство интегрируемости уравнений с локальными свойствами его решений [3]. С тех пор критерий Ковалевской-Пенлеве широко используется в современной теории интегрируемых систем как для построения новых примеров интегрируемых систем, так и для изучения их свойств. Из всего многообразия литературы по данному вопросу мы будем непосредственно использовать обзоры $[2,4,5]$ с учетом указанной в них литературы.

Дальнейшее развитие идей интегрируемости и хаоса привело к обобщению критериев интегрируемости и созданию слабого критерия Пенлеве и критерия поли-Пенлеве $[2,6]$. Напомним, что дифференциальные уравнения удовлетворяют критерию Ковалевской-Пенлеве, если их аналитические решения имеют в комплексной плоскости времени подвижные особые точки только типа полюса $\left(t-t_{0}\right)^{-m}, m$-целое. В то же время слабый критерий Пенлеве [2] допускает наличие подвижных особых точек типа

\footnotetext{
${ }^{*}$ Санкт-Петербургский государственный университет, Санкт-Петербург, Россия. E-mail:tsiganov@mph.niif.spb.su
} 
алгебраических точек разветвления $\left(t-t_{0}\right)^{1 / k}, k$ - целое. Очевидная замена переменной $\tau=\left(t-t_{0}\right)^{1 / k}$, которая зависит от начальных данных $t_{0}$, не всегда приводит к правильному разложению Пенлеве для всех аналитических решений уравнений движения одновременно [2]. Тем не менее с помошью замены времени вместе с подходящей заменой остальных зависимых от времени физических переменных довольно часто можно связать интегрируемые системы, удовлетворяющие слабому критерию Пенлеве, с системами, отвечающими полному критерию интегрируемости Ковалевской-Пенлеве. Заметим также, что замена времени является одним из основных инструментов качественной теории динамических систем, основы которой были заложены Пуанкаре и которая широко используется в конкретных задачах астрофизики и газовой динамики [4]. Однако в этом случае замена времени используется для решения конкретной задачи, а не для связи различных физических систем.

Преобразования, которые используют замену времени, являются заведомо неканоническими, и поэтому связанные таким образом интегрируемые динамические системы часто называют дуальнылми [2]. Подобная дуальность разных интегрируемых физических систем не нова. Так, в 1901 г. Колосов [7] обнаружил связь волчка Ковалевской с задачей о движении материальной точки в двумерной плоскости $(x, y)$ под действием некоторого потенциала. Задача на плоскости разделяется в обычных эллиптических координатах $\left\{q_{1}, q_{2}\right\}$, которые совпадают с переменными Ковалевской. Указанные две интегрируемые системы связаны неканоническим преобразованием времени вида

$$
d \tilde{t}=\left(q_{1}(t)+q_{2}(t)\right) d t
$$

Позднее с использованием подобных неканонических преобразований времени была установлена связь волчка Ковалевской с задачей о движении по геодезическим на группе $S O(4)$ [8] и с задачей Неймана на сфере $S^{2}$ [9].

Другим классическим примером является дуальность задачи Кеплера в $\mathbb{R}^{2 n}$ задаче о движении по геодезическим на сфере $S^{n}$ [10] или гармоническому осциллятору [11]. Несколько других примеров дуальности конечномерных интегрируемых систем приведено также в обзоре [2].

В данной работе рассматриваются дуальные штеккелевские системы, связанные неканоническим преобразованием переменной времени. При этом замена независимой переменной времени определяется неявно с помощью преобразования соответствующих функций Гамильтона в терминах переменных разделения. Следует подчеркнуть, что обычно методы сингулярного анализа используются для определения интегрируемости физической модели, заданной в конкретных физических переменных. Мы же берем заведомо интегрируемые системы и изучаем существующие между ними неканонические связи. Поэтому в основном мы будем заниматься исследованием свойств дуальных интегрируемых систем, построением пар Лакса и выгислением соответствующих $r$-матричных алгебр. При этом мы не будем детально рассматривать алгебро-геометрические аспекты задачи [5] и использовать непосредственно сам сингулярный метод [2]

В качестве примеров изучаются несколько систем с двумя степенями свободы, для которых разделение переменных не было известно ранее. Среди них - интегрируемые 
деформации задачи Кеплера, близкие к системам типа Драша [12], и системы типа Холта [2] со вторым интегралом движения третьей или четвертой степеней по импульсам. Для всех рассмотренных примеров неканоническоепреобразование времени будет близко к преобразованию Колосова (1.1) [7].

\section{2. ЗАМЕНА ВРЕМЕНИ ДЛЯ ШТЕККЕЛЕВСКИХ СИСТЕМ}

Рассмотрим голономные системы на фазовом пространстве $\mathbb{R}^{2 n}$ со стандартной симплектической формой $\Omega_{n}$ и обычными скобками Пуассона

$$
\Omega_{n}=\sum_{j=1}^{n} d p_{j} \wedge d q_{j}, \quad\left\{p_{j}, q_{k}\right\}=\delta_{j k},
$$

где $\left\{p_{j}, q_{j}\right\}_{j=1}^{n}$ - канонические переменные в $\mathbb{R}^{2 n}$.

Вполне интегрируемые штеккелевские системы [13] в $\mathbb{R}^{2 n}$ однозначно определяются невырожденной $(n \times n)$-матрищей Штеккеля $\mathbf{S}$, элементы $j$-го столбца которой $\mathrm{s}_{k j}$ зависят лишш от координаты $q_{j}$ :

$$
\operatorname{det} \mathbf{S} \neq 0, \quad \frac{\partial \mathrm{s}_{k j}}{\partial q_{m}}=0, \quad j \neq m .
$$

Данной матрице $\mathbf{S}$ сопоставим $n$ квадратичных по импульсам функционально независимых интегралов движения $I_{k}, k=1, \ldots, n$, находящихся в инволюшии $\left\{I_{k}, I_{j}\right\}=0$, по правилу

$$
I_{k}=\sum_{j=1}^{n} c_{j k}\left(p_{j}^{2}+U_{j}\left(q_{j}\right)\right), \quad c_{j k}=\frac{\mathrm{S}_{k j}}{\operatorname{det} \mathbf{S}} .
$$

Здесь $\mathbf{C}=\left[c_{j k}\right]$ - матриша, обратная к матрице Штеккеля $\mathbf{S}, \mathbf{S}_{k j}$ - алгебраическое дополнение элемента $\mathrm{s}_{k j}$ в определителе $\operatorname{det} \mathbf{S}$.

В качестве гамильтониана можно рассматривать любой из интегралов $I_{k}(2.2)$ с соответствуюшим сопряженным временем $t_{k}$, так что для любой функции $\xi(\mathrm{p}, \mathrm{q})$ справедливо равенство

$$
\frac{d \xi(\mathrm{p}, \mathrm{q})}{d t_{k}}=\left\{I_{k}, \xi(\mathrm{p}, \mathrm{q})\right\} .
$$

Для определенности мы выберем первый из интегралов $I_{1}$ в качестве функции Гамильтона $H$, которая сопряжена времени $t$.

Совместная поверхность уровня всех интегралов $(2.2)$

$$
M_{\alpha}=\left\{z \in \mathbb{R}^{2 n}: I_{k}(z)=\alpha_{k}, k=1, \ldots, n\right\}
$$

диффеоморфна $n$-мерному тору [14], откуда сразу же следует, что

$$
p_{j}^{2}=\left(\frac{\partial \mathcal{S}}{\partial q_{j}}\right)^{2}=\sum_{k=1}^{n} \alpha_{k} \mathrm{~s}_{k j}\left(q_{j}\right)-U_{j}\left(q_{j}\right),
$$


где $\mathcal{S}\left(q_{1}, \ldots, q_{n}\right)$ - функция укороченного действия [14]. Поэтому для рассматриваемого случая уравнение Гамильтона-Якоби на $M_{\alpha}$

$$
\frac{\partial \mathcal{S}}{\partial t}+H\left(t, \frac{\partial \mathcal{S}}{\partial q_{1}}, \ldots, \frac{\partial \mathcal{S}}{\partial q_{n}}, q_{1}, \ldots, q_{n}\right)=0 \quad \Rightarrow \quad c_{j 1} \frac{\partial \mathcal{S}}{\partial q_{j}} \frac{\partial \mathcal{S}}{\partial q_{j}}=E
$$

допускает разделение переменных

$$
\mathcal{S}\left(q_{1}, \ldots, q_{n}\right)=\sum_{j=1}^{n} \mathcal{S}_{j}\left(q_{j}\right), \quad \mathcal{S}_{j}\left(q_{j}\right)=\int \sqrt{F_{j}\left(q_{j}\right)} d q_{j},
$$

где функции $F_{j}(\lambda)$ зависят от $n$ параметров $\left\{\alpha_{k}\right\}_{k=1}^{n}$ :

$$
F_{j}(\lambda)=\sum_{k=1}^{n} \alpha_{k} \mathrm{~s}_{k j}(\lambda)-U_{j}(\lambda)
$$

Согласно стандартной процедуре интегрирования уравнения Гамильтона-Якоби в квадратурах величины $q_{j}\left(t, \alpha_{1}, \ldots, \alpha_{n}\right)$ находятся из уравнения, включаюшего явно время,

$$
\sum_{j=1}^{n} \int_{\gamma_{0}\left(p_{0}, q_{0}\right)}^{\gamma_{j}\left(p_{j}, q_{j}\right)} \frac{\mathrm{s}_{1 j}(\lambda) d \lambda}{\sqrt{\sum_{k=1}^{n} \alpha_{k} \mathrm{~s}_{1 j}(\lambda)-U_{j}(\lambda)}}=\beta_{1}=t
$$

и остальных $n-1$ уравнений, не зависяших явно от времени,

$$
\sum_{j=1}^{n} \int_{\gamma_{0}\left(p_{0}, q_{0}\right)}^{\gamma_{j}\left(p_{j}, q_{j}\right)} \frac{\mathrm{s}_{k j}(\lambda) d \lambda}{\sqrt{\sum_{k=1}^{n} \alpha_{k} \mathrm{~s}_{k j}(\lambda)-U_{j}(\lambda)}}=\beta_{k}, \quad k=2, \ldots, n
$$

Таким образом, теорема Штеккеля [13] полностью решает вопрос о разделении переменных для систем с гамильтонианами вида (2.10) (см. ниже).

Перейдем теперь к рассмотрению неканонических преобразований, связывающих различные интегрируемые системы с функциями Гамильтона типа Штеккеля (2.10) (см. ниже).

ПРЕДЛОЖЕниЕ 1. Любъе две матрици Штеккеля $\mathbf{S}$ и $\widetilde{\mathbf{S}}$, которые отличаются друг от друга только первой строкой

$$
\mathrm{s}_{k j}=\tilde{\mathrm{s}}_{k j}, \quad k \neq 1,
$$

определяют дуальные интегрируемые системы с гамильтонианами $H$ и $\widetilde{H}$ :

$$
\widetilde{H}=v(q) H, \quad \text { əдe } \quad v(q)=\frac{\operatorname{det} \mathbf{S}\left(q_{1}, \ldots, q_{n}\right)}{\operatorname{det} \widetilde{\mathbf{S}}\left(q_{1}, \ldots, q_{n}\right)}
$$


отношение которых равно отношению определителей данных матрии Штеккеля.

Действительно, уравнение (2.9) непосредственно следует из определения гамильтониана

$$
H=\sum_{j=1}^{n} c_{j 1}\left(p_{j}^{2}+U_{j}\left(q_{j}\right)\right)
$$

и элементов обратной матрицы

$$
c_{j 1}=\frac{\mathrm{S}_{1 j}}{\operatorname{det} \mathbf{S}}=\frac{1}{\operatorname{det} \mathbf{S}} \frac{\partial \operatorname{det} \mathbf{S}}{\partial \mathrm{s}_{1 j}} .
$$

Таким образом, соотношение (2.9) задает замену времени $t \rightarrow \tilde{t}$, где $t$ и $\tilde{t}$ сопряжены интегралам $H=I_{1}$ и $\widetilde{H}=\tilde{I}_{1}$, соответственно. Уравнение $(2.7)$, которое содержит элементы первой строки матриц Штеккеля $\mathbf{S}$ и $\widetilde{\mathbf{S}}$ и переменные времени $t$ и $\tilde{t}$, можно рассматривать как определяющее преобразование $t \rightarrow \tilde{t}$.

Таким образом, все множество штеккелевских систем в $\mathbb{R}^{2 n}$ можно разбить на семейства дуальных систем, связанных неканоническим преобразованием. Более того, заменяя одну строку за другой, мы можем построить последовательность неканонических преобразований времен $t_{k} \rightarrow \tilde{t}_{k}, k=1, \ldots, n$, которая связывает данную штеккелевскую систему с любой другой штеккелевской системой в $\mathbb{R}^{2 n}$.

В качестве примера рассмотрим три матрищы Штеккеля вида

$$
\mathbf{S}=\left(\begin{array}{cc}
1 & 1 \\
1 & -1
\end{array}\right), \quad \widetilde{\mathbf{S}}=\left(\begin{array}{cc}
q_{1} & q_{2} \\
1 & -1
\end{array}\right), \quad \widehat{\mathbf{S}}=\left(\begin{array}{cc}
q_{1}^{2} & q_{2}^{2} \\
1 & -1
\end{array}\right)
$$

Соответствуюшие гамильтонианы $H(2.10), \widetilde{H}$ и $\widehat{H}$ дуальны:

$$
\begin{aligned}
\widetilde{H} & =\frac{\left(q_{1}+q_{2}\right)^{-1}}{2} H, \\
\widehat{H} & =\frac{\left(q_{1}^{2}+q_{2}^{2}\right)^{-1}}{2} H=\frac{q_{1}+q_{2}}{q_{1}^{2}+q_{2}^{2}} \widetilde{H},
\end{aligned}
$$

при любом значении потенциала $U$. Для двух дуальных гамильтонианов $\widetilde{H}$ и $\widehat{H}$ замена переменной времени в случае (2.12) близка к преобразованию Колосова (1.1) [7], т.к. функция $v$ в (2.9) зависит только от координат и для любой другой функции от координат $\xi(q)$ справедливо уравнение

$$
\frac{d \xi(\mathrm{q})}{d \tilde{t}}=\{\widetilde{H}, \xi(\mathrm{q})\}=\frac{\left(q_{1}+q_{2}\right)^{-1}}{2}\{H, \xi(\mathrm{q})\}=\frac{1}{2\left(q_{1}+q_{2}\right)} \frac{d \xi(\mathrm{q})}{d t} .
$$

Например, рассмотрим дуальные системы с однородным кубическим потенциалом вида

$$
U\left(q_{j}\right)=2 \alpha^{2} q_{j}^{3}+\beta q_{j}^{2}+\gamma q_{j}+\delta
$$


В переменных разделения гамильтониан $H$ в явном виде равен

$$
H=\frac{1}{4}\left(p_{1}^{2}+p_{2}^{2}\right)+\alpha^{2}\left(q_{1}^{3}+q_{2}^{3}\right)+\frac{\beta}{2}\left(q_{1}^{2}+q_{2}^{2}\right)+\frac{\gamma}{2}\left(q_{1}+q_{2}\right)+\delta
$$

Перейдем к физическим координатам в первом случае по правилу

$$
\begin{array}{ll}
q_{1}=\frac{x+y}{2}, & p_{1}=p_{x}+p_{y} \\
q_{2}=\frac{x-y}{2}, & p_{2}=p_{x}-p_{y}
\end{array}
$$

во втором случае по правилу

$$
\begin{array}{ll}
q_{1}=\frac{3}{4} x^{2 / 3}+\frac{p_{y}}{3 \alpha}, & p_{1}=p_{x} x^{1 / 3}-\frac{3 \alpha}{2} y \\
q_{2}=\frac{3}{4} x^{2 / 3}-\frac{p_{y}}{3 \alpha}, & p_{2}=p_{x} x^{1 / 3}+\frac{3 \alpha}{2} y
\end{array}
$$

и в третьем случае по правилу

$$
\begin{array}{ll}
q_{1}=\sqrt{x}-\sqrt{y}, & p_{1}=p_{x} \sqrt{x}-p_{y} \sqrt{y} \\
q_{2}=-i(\sqrt{x}+\sqrt{y}), & p_{2}=i\left(p_{x} \sqrt{x}+p_{y} \sqrt{x}\right) .
\end{array}
$$

В новых переменных все функции Гамильтона (2.12), (2.15) приобретают натуральный вид, если наложить ограничение $\beta=0$ на потенциал $U(2.14)$ для второго гамильтониана $\widetilde{H}$. Выпишем в явном виде функции Гамильтона для некоторых частных значений коэффициентов потенциала:

$$
\begin{array}{rlrl}
H & =\frac{1}{2}\left(p_{x}^{2}+p_{y}^{2}\right)+\frac{\alpha^{2}}{4} x\left(x^{2}+3 y^{2}\right)+\frac{\beta}{4}\left(x^{2}+y^{2}\right)+\frac{\gamma}{2} x+\delta, \\
\widetilde{H} & =\frac{1}{2}\left(p_{x}^{2}+p_{y}^{2}\right)+\frac{9 \alpha^{2}}{8} x^{-2 / 3}\left(\frac{3}{4} x^{2}+y^{2}\right)+\delta x^{-2 / 3}+\frac{3 \gamma}{4} \quad \text { при } \beta=0, \\
\widehat{H} & =\frac{1}{2} p_{x} p_{y}-\frac{\beta}{2} \frac{1}{\sqrt{x y}}+\frac{\gamma}{4}\left(\frac{1+i}{\sqrt{x}}-\frac{1-i}{\sqrt{y}}\right)+\delta & \text { при } \quad \alpha=0 .
\end{array}
$$

Дуальность первой и третьей систем при $\alpha=0$ - это хорошо известная дуальность гармонического осциллятора и задачи Кеплера [11]. При $\alpha \neq 0$ система с гамильтонианом $H$ известна как один из интегрируемых случаев Энона-Эйлеса [2], а вторая система с гамильтонианом $\widetilde{H}$ известна как система Холта [2]. Дуальность этих двух систем была замечена ранее [2], хотя для системы типа Холта переменные разделения $\left\{p_{j}, q_{j}\right\}_{j=1}^{2}$ известны не были. При этом каноническое преобразование для системы Холта устроено таким образом, что соответствующий второй интеграл движения является многочленом третьей степени по импульсам. 
Даже на этом простом примере видно, что основной проблемой подхода Штеккеля к конструированию интегрируемых систем является переход от переменных разделения к физическим переменным, например к переменным $\left\{p_{j}, x_{j}\right\}_{j=1}^{n}$, в терминах которых гамильтониан приобретает натуральный вид и уравнения движения становятся уравнениями Ньютона:

$$
H=T+V\left(x_{1}, \ldots, x_{n}\right), \quad \ddot{x}_{j}=\Phi_{j}\left(x_{1}, \ldots, x_{n}\right) .
$$

Частично данная проблема решена для однородных штеккелевских систем при $U_{j}=U$, $j=1, \ldots, n$, с введением пары Лакса [15]. При этом следует подчеркнуть, что точки ветвления в комплексной плоскости появляются для решений уравнений движения, заданных именно в терминах физических переменных.

Далее мы рассмотрим подробнее несколько других примеров дуальности, построим представления Лакса и линейные $r$-матричные алгебры для некоторых однородных систем, удовлетворяюших слабому критерию Пенлеве после перехода к физическим переменным.

\section{3. ДУАЛЬНОСТЬ И ОТОБРАЖЕНИЕ АБЕЛЯ}

Всюду далее будут рассматриваться однородные штеккелевские системы с однородными полиномиальными потенциалами $U_{j}(q)=U, j=1, \ldots, n$. В этом случае задача об интегрировании уравнений движения сводится к стандартной задаче алгебраической геометрии [15]. Напомним несколько фактов об отображении Абеля и задаче обрашения Якоби на гиперэллиптических кривых.

Пусть $\mathcal{C}(z, \lambda)$ - гиперэллиптическая кривая рода $g$, например вида

$$
\mathcal{C}: z^{2}=F(\lambda)=\sum_{k=0}^{2 g+1} e_{k} \lambda^{k}=\prod_{j=1}^{2 g+1}\left(\lambda-\lambda_{j}\right),
$$

где $F(\lambda)$ - многочлен без кратных корней, а числа $e_{k}$ и $\lambda_{j}$ - модули кривой $\mathcal{C}$. Определив абелеву группу дивизоров $\operatorname{Div}(\mathcal{C})$ и якобиан $J(\mathcal{C})$ алгебраической кривой $\mathcal{C}$, введем отображение Абеля [16, 17]

$$
\mathcal{U}: \operatorname{Div}(\mathcal{C}) \rightarrow J(\mathcal{C})
$$

Неупорядоченные наборы из $n$ точек поверхности $\mathcal{C}$ образуют $n$-ю симметрическую степень $\mathcal{C}^{(n)}=S^{n} \mathcal{C}$ римановой поверхности $\mathcal{C}$. Рассмотрим ограничение отображения Абеля (3.2) на $\mathcal{C}^{(n)}$

$$
\mathcal{U}: \mathcal{C}^{(n)} \rightarrow J(\mathcal{C})
$$

где

$$
\mathcal{U}\left(\gamma_{1}, \gamma_{2}, \ldots, \gamma_{n}\right)=\mathcal{U}\left(\gamma_{1}\right)+\mathcal{U}\left(\gamma_{2}\right)+\cdots+\mathcal{U}\left(\gamma_{n}\right)
$$

Если степень $n$ равна роду кривой $g=n$, то отображение $\mathcal{U}(3.3)$ однозначно $[16,17]$. Подчеркнем, что далее мы будем изучать случай с $g \geqslant n$, когда отображение Абеля неоднозначно, это и приводит к появлению дуальных интегрируемых систем.

2 Теоретическая и математическая физика, т. 120, № 1, 1999 г. 
Предположим, что точка $D=\gamma_{1}+\cdots+\gamma_{n}$ принадлежит пространству $\mathcal{C}^{(n)}$ при $g \geqslant n$. Производная отображения Абеля (3.3) в точке $D$ есть линейное отображение из касательного пространства $T_{D}\left(\mathcal{C}^{(n)}\right)$ к многообразию $\mathcal{C}^{(n)}$ в точке $D$ в касательное пространство $T_{\mathcal{U}(D)}(J(\mathcal{C}))$ якобиана $J(\mathcal{C})$ в точке $\mathcal{U}(D)$ :

$$
\mathcal{U}_{D}^{*}: T_{D}\left(C^{(n)}\right) \rightarrow T_{\mathcal{U}(D)}(J(\mathcal{C}))
$$

Если $D$ - дивизор обшего положения и координаты $x_{j}$ на кривой $\mathcal{C}$ в окрестности точек $\gamma_{j}$ образуют систему локальных координат $\left(x_{1}, \ldots, x_{n}\right)$ в окрестности точки $D$ в $\mathcal{C}^{(n)}$, то канонический базис $d \mathrm{w}_{k}(k=1, \ldots, g)$ в пространстве голоморфных дифференциалов $\mathcal{H}_{1}(\mathcal{C})$ имеет вид

$$
d \mathrm{w}_{k}=\varphi_{k j}\left(x_{j}\right) d x_{j},
$$

где функции $\phi_{k j}\left(x_{j}\right)$ голоморфны. Отображение Абеля $\mathcal{U}$ в данной окрестности точки $D$ равно

$$
\mathcal{U}\left(x_{1}, \ldots, x_{k}\right)=\left(\sum_{j=1}^{n} \int_{\gamma_{0}}^{x_{j}} \varphi_{1 j}\left(x_{j}\right) d x_{j}, \ldots, \sum_{j=1}^{n} \int_{\gamma_{0}}^{x_{j}} \varphi_{g j}\left(x_{j}\right) d x_{j}\right) .
$$

Следовательно, производная отображения Абеля [18] имеет вид

$$
\mathcal{U}_{D}^{*}=\left(\begin{array}{ccc}
\varphi_{11}\left(\gamma_{1}\right) & \cdots & \varphi_{g 1}\left(\gamma_{1}\right) \\
\vdots & \ddots & \vdots \\
\varphi_{1 n}\left(\gamma_{n}\right) & \cdots & \varphi_{g n}\left(\gamma_{n}\right)
\end{array}\right)
$$

Матрица $\mathcal{U}_{D}^{*}$ размерности $(n \times g)$ называется матрицей Бриля-Нетера.

Всюду далее мы будем рассматривать симметрические дивизоры $D_{s}$, для которых коэффициенты разложения дифференциалов (3.4) не зависят от номера точки $\gamma_{j}$,

$$
d \mathrm{w}_{k}=\phi_{k}\left(x_{j}\right) d x_{j} .
$$

В этой точке строки матрицы Бриля-Нетера имеют одинаковую функциональную зависимость от локальных координат $\left\{x_{1}, \ldots, x_{n}\right\}$. Назовем такие матрицы $\mathcal{U}_{D}^{*}$ симметрическими матрицами Бриля-Нетера.

Задача обрашения отображения Абеля $\mathcal{U}$ (3.3) ставится так (задача обрашения Якоби (2.8)): для данной точки $\mathbf{u}=\left(\beta_{1}, \beta_{2}, \ldots, \beta_{n}\right) \in J(\mathcal{C})$ найти $n$ точек $\gamma_{1}, \gamma_{2}, \ldots, \gamma_{n}$ римановой поверхности $\mathcal{C}$ рода $g$ таких, что

$$
\sum_{k=1}^{g} \int_{\gamma_{0}}^{\gamma_{k}} d \mathrm{w}_{j}=\beta_{j}, \quad j=1, \ldots, n .
$$

Здесь $d \mathrm{w}_{j}$ - канонический базис голоморфных дифференциалов кривой $\mathcal{C}, \gamma_{0}$ - подходящим образом выбранная базовая точка поверхности $\mathcal{C}[16]$.

Известно [16], что для почти любой точки $\mathbf{u} \in J(\mathcal{C})$ решение $D=\gamma_{1}+\cdots+\gamma_{n}$ существует и определяется системой (3.6) однозначно (без учета порядка точек $\gamma_{j}$ ), только 
если степень $n$ равна роду кривой $g$. Если число $n$ меншше рода кривой $g$, то отображение Абеля неоднозначно и двум точкам $\mathbf{u}, \tilde{\mathbf{u}} \in J(\mathcal{C})$ может отвечать один прообраз отображения Абеля $\left\{\gamma_{1}, \ldots, \gamma_{n}\right\} \in \mathcal{C}^{(n)}$.

Сушествует несколько методов решения задачи обрашения Якоби [16]. При рассмотрении штеккелевских систем нам будет удобно комбинировать подход Нетера [18], который мы уже частично использовали, и подход Больца [19]. Так, в соответствии с работами $[17,19]$ из уравнений $(2.7),(2.8)$ координаты $\left\{q_{1}, \ldots, q_{n}\right\}$ могут быть определены как решения первого уравнения Больца:

$$
e(\lambda, \mathbf{u})=\lambda^{n}-\lambda^{n-1} \wp_{n, n}(\mathbf{u})-\lambda^{n-2} \wp_{n, n-1}(\mathbf{u})-\cdots-\wp_{n, 1}(\mathbf{u})=0
$$

а сопряженные им импульсы $\left\{p_{1}, \ldots, p_{n}\right\}$ определяются вторым уравнением Больца

$$
p_{k}=-\left.\frac{\partial e(\lambda, \mathbf{u})}{\partial \beta_{n}}\right|_{\lambda=q_{k}} .
$$

Здесь $\mathbf{u} \in J(\mathcal{C})$ - точка, принадлежашая якобиану кривой $\mathcal{C}$, а $\wp k, j(\mathbf{u})$ - клейновские ю-функции $[17,19]$. При этом канонически-сопряженные пары переменных разделения $\left\{\left(p_{1}, q_{1}\right), \ldots,\left(p_{n}, q_{n}\right)\right\} \in \mathcal{C}^{n}$ задают дивизор общего положения $D=\gamma_{1}+\cdots+\gamma_{n}$, $\operatorname{deg} D=n$. Заметим, что в общем случае функцию $e(\lambda, \mathbf{u})$, которая имеет $g$ нулей, даюших решение задачи обрашения Якоби, можно переписать в виде

$$
e(\lambda, \mathbf{u})=\theta\left(\mathcal{U}\left(\gamma_{1}, \ldots, \gamma_{g}\right)-\mathbf{u}-K\right), \quad \mathcal{U}\left(\gamma_{1}, \ldots, \gamma_{g}\right)=\mathcal{U}\left(\gamma_{1}\right)+\cdots+\mathcal{U}\left(\gamma_{g}\right)
$$

где $K$ - вектор римановых констант [16].

Применим эту теорию к штеккелевским системам [13]. Действительно, для однородных штеккелевских систем каждое выражение (2.4) можно рассматривать как риманову поверхность

$$
\mathcal{C}: z^{2}=F(\lambda), \quad F(\lambda)=\sum_{k=1}^{n} \alpha_{k} \mathrm{~s}_{k j}(\lambda)-U(\lambda),
$$

некоторого рода $g$. Заметим, что для системы на фазовом пространстве $\mathbb{R}^{2 n}$ минимально допустимьй род $g$ кривой $\mathcal{C}$ равен $[(n-1) / 2]$.

Симметрическая степень $\mathcal{C}^{(n)}=S^{n} \mathcal{C}$ римановой поверхности $\mathcal{C}$ задает $n$-мерное лагранжево подмногообразие полного симплектического многообразия

$$
\mathcal{C}^{(n)}: \mathcal{C}\left(p_{1}, q_{1}\right) \times \mathcal{C}\left(p_{2}, q_{2}\right) \times \cdots \times \mathcal{C}\left(p_{n}, q_{n}\right)
$$

где импульсы находятся из уравнения (2.4). При этом задача интегрирования уравнений движения (2.7), (2.8) сводится к задаче обращения Якоби (3.2) на лагранжевом многообразии (3.11). Соответствуюшие голоморфные дифференциалы имеют стандартный вид $[16]$

$$
d \mathrm{w}_{k}=\frac{\mathrm{s}_{k j}(\lambda) d \lambda}{z(\lambda)} .
$$


Множество данных дифференциалов либо является полным базисом в пространстве голоморфных дифференциалов $\mathcal{H}_{1}(\mathcal{C})$, либо может быть дополнено до него. Соответствующая матрица Штеккеля $\mathbf{S}$ определяется $(n \times n)$-блоком транспонированной $(g \times n)$-матрицы Бриля-Нетера $\mathcal{U}_{D}^{*}$ при $g \geqslant n$.

Различные блоки матрищы Бриля-Нетера отвечают различным интегрируемым системам, которые могут быть дуальны, (2.9). При этом для двух дуальных систем соответствуюшие дифференциалы (3.12) не образуют полного базиса в $\mathcal{H}_{1}(\mathcal{C})$ на единой для них кривой $\mathcal{C}$. В силу этого двум точкам якобиана, отличающимся первой компонентой,

$$
\mathbf{u}=\left\{t, \beta_{2}, \ldots, \beta_{n}\right\} \in J(\mathcal{C}) \quad \text { и } \quad \tilde{\mathbf{u}}=\left\{\tilde{t}, \beta_{2}, \ldots, \beta_{n}\right\} \in J(\mathcal{C})
$$

отвечает один прообраз отображения Абеля $\left\{\left(p_{1}, q_{1}\right), \ldots,\left(p_{n}, q_{n}\right)\right\} \in \mathcal{C}^{(n)}$.

Таким образом, мы можем сделать вывод о взаимосвязи неоднозначности отображения Абеля с сушествованием неканонического преобразования времени, связывающего различные интегрируемые системы.

\section{4. ПРЕДСТАВЛЕНИЕ ЛАКСА}

В общем случае представление Лакса для штеккелевских систем неизвестно. Тем не менее для ряда важных физических примеров матрицы Лакса можно построить и, более того, можно предложить единый подход к построению данных матриц.

Всюду далее мы ограничимся стандартным базисом в пространстве голоморфных дифференциалов $\mathcal{H}_{1}(\mathcal{C})$

$$
d \mathrm{w}_{j}=\frac{\lambda^{j-1}}{z(\lambda)} d \lambda, \quad j=1, \ldots, g .
$$

Используя соответствующую симметрическую матрицу Бриля-Нетера $\mathcal{U}_{D}^{*}$, определим матрицу Штеккеля как $(n \times n)$-блок матрищы размерности $(g \times n)$ :

$$
\left(\begin{array}{cccc}
q_{1}^{g-1} & q_{2}^{g-1} & \cdots & q_{n}^{g-1} \\
q_{1}^{g-2} & q_{2}^{g-2} & \cdots & q_{n}^{g-2} \\
\vdots & \ddots & \vdots & \vdots \\
1 & 1 & \cdots & 1
\end{array}\right)
$$

Как видно, не все матрицы Штеккеля могут быть получены из симметрических матриц Бриля-Нетера. Например, уже рассмотренные нами матрицы (2.11) не принадлежат семейству симметрических матриц.

Для $(n \times n)$-матриц Штеккеля $\mathbf{S}$, полученных из матрицы (4.2), будем искать представление Лакса для штеккелевских систем в виде

$$
L=\left(\begin{array}{cc}
h(\lambda, \mathrm{p}, \mathrm{q}) & e(\lambda, \mathrm{q}) \\
f(\lambda, \mathrm{p}, \mathrm{q}) & -h(\lambda, \mathrm{p}, \mathrm{q})
\end{array}\right) .
$$

Далее для краткости мы будем опускать некоторые аргументы элементов матрицы Лакca. 
Теперь выберем гиперэллиптическую кривую $\mathcal{C}$ рода $g$ и размерность фазового пространства $n \leqslant g$. Выделим из матрицы $(4.2)(n \times n)$-блок-матрицу Штеккеля $\mathbf{S}$ и построим по ней функцию Гамильтона $H(2.10)$ с нулевым потенциалом $U=0$. Затем определим функцию $e(\lambda, \mathbf{u})(3.7)$,

$$
e(\lambda, \mathrm{q})=\prod_{j=1}^{n}\left(\lambda-q_{j}\right)
$$

нули которой дают решение задачи обрашения Якоби (2.8).

На следуюшем шаге определим второй элемент матрищы Лакса

$$
h(\lambda, \mathrm{p}, \mathrm{q})=-\frac{1}{2 v(\lambda, \mathrm{q})} \frac{d e(\lambda, \mathrm{q})}{d t}+w(\lambda, \mathrm{p}, \mathrm{q}) e(\lambda, \mathrm{q})
$$

Здесь функция координат $v(\lambda, \mathrm{q})$ определяется из уравнения

$$
\left.h(\lambda)\right|_{\lambda=q_{k}}=p_{k}=\left.\left(\frac{1}{2 v} \frac{d e(\lambda)}{d t}\right)\right|_{\lambda=q_{k}}=-\left.\frac{\partial e(\lambda)}{\partial \beta_{n}}\right|_{\lambda=q_{k}}
$$

с учетом второго уравнения Больца (3.8). Третий элемент матрищы Лакса определим из уравнения

$$
f(\lambda)=\frac{1}{v} \frac{d h(\lambda)}{d t} .
$$

Единственная не определенная пока функция $w(\lambda, \mathrm{p}, \mathrm{q})$ выбирается таким образом, чтобы спектральная кривая матрицы Лакса (4.3)

$$
\mathcal{C}: z^{2}=F(\lambda)=-\operatorname{det} L_{0}(\lambda)=h^{2}(\lambda)+e(\lambda) f(\lambda)
$$

совпадала с исходной алгебраической кривой $\mathcal{C}(2.4)$ при $U=0$.

Построенная таким образом матрица Лакса $L_{0}(\lambda)(4.3)$ :

$$
L_{0}(\lambda)=\left(\begin{array}{cc}
-\frac{1}{2 v} e_{t}(\lambda)+w(\lambda, \mathrm{p}, \mathrm{q}) e(\lambda) & e(\lambda) \\
\frac{1}{v} h_{t}(\lambda) & \frac{1}{2 v} e_{t}(\lambda)-w(\lambda, \mathrm{p}, \mathrm{q}) e(\lambda)
\end{array}\right),
$$

где

$$
e_{t}=\frac{d e(\lambda)}{d t}=\{H, e(\lambda)\}, \quad h_{t}=\frac{d h(\lambda)}{d t}=\{H, h(\lambda)\},
$$

удовлетворяет уравнению Лакса

$$
\frac{d L_{0}}{d t}=\left\{H, L_{0}\right\}=\left[A_{0}, L_{0}\right]
$$

с матрищей

$$
A_{0}=v(\lambda, \mathrm{q})\left(\begin{array}{cc}
w(\lambda, \mathrm{p}, \mathrm{q}) & 1 \\
0 & -w(\lambda, \mathrm{p}, \mathrm{q})
\end{array}\right)
$$


По определению матрищы $L_{0}(\lambda)(4.7)$ пары сопряженных переменных разделения $\gamma_{j}=$ $\left(p_{j}, q_{j}\right)(4.4),(4.5)$ расположены на спектральной кривой $\mathcal{C}(4.6)$ матришы Лакса

$$
z^{2}\left(\gamma_{j}\right)=p_{j}^{2}=\left.h^{2}(\lambda)\right|_{\lambda=q_{j}}=F\left(\lambda=q_{j}\right)=\left.F(\lambda)\right|_{\gamma_{j}}
$$

Для систем с полиномиальным потенциалом $U \neq 0$ предлагается изменить элемент $f(\lambda)$ в матрице (4.7) по правилу

$$
f(\lambda)=\frac{1}{v} \frac{d h(\lambda)}{d t}+u(\lambda, \mathrm{q}) e(\lambda)
$$

добавив новую функцию координат $u(\lambda, \mathrm{q})$. Соответствуюшая матрица Лакса имеет вид

$$
L(\lambda)=\left(\begin{array}{cc}
-\frac{1}{2 v} e_{t}(\lambda)+w(\lambda, \mathrm{p}, \mathrm{q}) e(\lambda) & e(\lambda) \\
\frac{1}{v} h_{t}(\lambda)+u(\lambda, \mathrm{q}) e(\lambda) & \frac{1}{2 v} e_{t}(\lambda)+w(\lambda, \mathrm{p}, \mathrm{q}) e(\lambda)
\end{array}\right)
$$

где производная по времени сопряжена уже гамильтониану с $U \neq 0$. Так как к гамильтониану добавляется потенциал, который зависит только от координат, то производная по времени элемента $e(\lambda)$ не изменяется, а вторая матрица $A$ изменяется по правилу

$$
A=A_{0}+\left(\begin{array}{cc}
0 & 0 \\
v(\lambda, \mathrm{q}) u(\lambda, \mathrm{q}) & 0
\end{array}\right)=v(\lambda, \mathrm{q})\left(\begin{array}{cc}
w(\lambda, \mathrm{p}, \mathrm{q}) & 1 \\
u(\lambda, \mathrm{q}) & -w(\lambda, \mathrm{p}, \mathrm{q})
\end{array}\right)
$$

Рассматривая соответствующее уравнение Лакса

$$
\frac{d L(\lambda)}{d t}=[A(\lambda), L(\lambda)]
$$

можно предположить, что общий множитель $v(\lambda, \mathrm{q})$ в матрице $A$ может быть связан с заменой времени для штеккелевских систем.

В общем случае для решения вопроса о сушествовании трех функций $v, w$ и $u$ необходимо использовать методы алгебраической геометрии [17], что выходит за рамки данной работы. В силу определения матрищ Лакса $L(\lambda)$ и $A(\lambda)$ эту задачу можно свести к доказательству сушествования решений $u(\lambda, \mathrm{q}), v(\lambda, \mathrm{q})$ и $w(\lambda, \mathrm{p}, \mathrm{q})$ одного уравнения

$$
\frac{d f(\lambda)}{d t}-2 v(u h-w f)=0 \quad \Longleftrightarrow \quad \frac{d F(\lambda, e, v, u)}{d t}=0
$$

для данной функции $e(\lambda)(4.4)$ и данного гамильтониана $H(2.10)$.

Для выделенной матрищы Штеккеля, которая отвечает нижнему блоку матрищы (4.2), отображение Абеля однозначно. В этом случае с помошью уравнений (3.8) и (4.5) можно доказать, что две функции тривиальны:

$$
v_{t}(\lambda, \mathrm{q})=0, \quad w(\lambda, \mathrm{p}, \mathrm{q})=0 \text {. }
$$


При этом, положив $t=x$ и $v(\lambda)=1$, можно переписать уравнение (4.9) в виде

$$
\frac{\partial u(x, \tau, \lambda)}{\partial \tau}=\left[\frac{1}{4} \partial_{x}^{3}+u(\lambda) \partial_{x}+\frac{1}{2} u_{x}(\lambda)\right] \cdot e(\lambda)=0 \text { при } x=t .
$$

Данное уравнение можно отождествить с уравнением $\partial u(x, \tau, \lambda) / \partial \tau=0$ для конечнозонных стационарных решений нелинейных эволюционных уравнений, где $\tau$ - новая переменная времени. При различном выборе функций $e(\lambda)$ и $u(\lambda, q)$ данная процедура приводит к различным иерархиям нелинейных уравнений, например к уравнению Кортевега-де Фриза, нелинейному уравнению Шредингера и уравнению синус-Гордон (см. ссылки в [15]).

При $v_{t}=0$ функция $u$ в (4.10) может быть получена из функции $e(\lambda)(3.7),(4.4)$ по правилу

$$
u(\lambda, \mathrm{q})=\left[\phi(\lambda) e^{-2}(\lambda, \mathrm{q})\right]_{N} .
$$

Здесь параметрическая функция $\phi(\lambda)$ - произвольная функция спектрального параметра $\lambda$ и символ $[\xi]_{N}$ - проекция элемента $\xi$ на $\mathrm{ad}_{R}^{*}$-инвариантные пуассоновы подпростанства соответствующей линейной $r$-матричной алгебры. Так, для рациональной $r$-матрицы данные проекции являются линейными комбинациями тейлоровских проекций

$$
[\xi]_{N}=\left[\sum_{k=-\infty}^{+\infty} \xi_{k} \lambda^{k}\right]_{N} \equiv \sum_{k=0}^{N} \xi_{k} \lambda^{k}
$$

или лорановских проекций $[15,20]$.

Хотя вопрос о справедливости данной конструкции представления Лакса для матрицы Бриля-Нетера обшего вида остается открытым, тем не менее данная конструкция подтверждается несколькими примерами штеккелевских систем, наиболее известными из физики. Так, все гамильтонианы натурального вида с рациональными потенциалами, допускаюшие точечное разделение переменных в стандартных криволинейных координатах, могут быть рассмотрены в рамках предложенного подхода [15]. Однако в этом случае $v_{t}(\lambda, q)=0$ и $w=0$, что достаточно сильно упрошает конструкцию представления Лакса и соответствующих $r$-матричных алгебр. Напомним, что в этом случае представления Лакса могут быть получены обычными алгебро-геометрическими методами $[15,21]$, а саму матрицу Лакса можно отождествить с элементом пополненной алгебры петель $\mathcal{L}(s l(2))$ в фундаментальном представлении.

В следуюшем разделе будут рассмотрены штеккелевские системы, дуальные к системам, допускающим точечное разделение переменных в стандартных криволинейных координатах. В этом случае функция $v(\lambda, \mathrm{q})$ равна отношению определителей соответствуюших матриц Штеккеля (2.9). Действительно, применяя соотношение (2.13) к функции координат $e(\lambda)(4.4)$ и используя определение (4.5), получим

$$
\begin{aligned}
\tilde{p}_{k} & =\left.\tilde{h}(\lambda)\right|_{\lambda=q_{k}}=\left(-\frac{1}{2 \tilde{v}}\{\widetilde{H}, e(\lambda)\}\right)_{\lambda=q_{k}}=\frac{\operatorname{det} \mathbf{S}}{\operatorname{det} \widetilde{\mathbf{S}}}\left(-\frac{1}{2 \tilde{v}}\{H, e(\lambda)\}\right)_{\lambda=q_{k}}= \\
& =\frac{\operatorname{det} \mathbf{S}}{\operatorname{det} \widetilde{\mathbf{S}}}\left(\frac{v}{\tilde{v}} h(\lambda)\right)_{\lambda=q_{k}}=p_{k} \frac{\operatorname{det} \mathbf{S}}{\operatorname{det} \widetilde{\mathbf{S}}}\left(\frac{v(\lambda, \mathrm{q})}{\tilde{v}(\lambda, \mathrm{q})}\right)_{\lambda=q_{k}} .
\end{aligned}
$$


Если отображение Абеля однозначно, то $v=1$ для одной из дуальных систем. Таким образом, в силу (4.13) мы в дальнейшем будем изучать штеккелевские системы со следуюшей функцией:

$$
v(\mathrm{q})=\frac{\operatorname{det} \mathbf{S}\left(q_{1}, \ldots, q_{n}\right)}{\operatorname{det} \widetilde{\mathbf{S}}\left(q_{1}, \ldots, q_{n}\right)} .
$$

Заметим, что именно эта функция определяет замену времени (2.9)-(2.13) типа Колосова (1.1).

Напомним, что для штеккелевских систем одной из главных задач является переход от переменных разделения к физическим переменным. Однако если отображение Абеля однозначно, то, положив $v=1$, можно определить новую функцию (см. ссылки в [15])

$$
\mathcal{B}^{2}(\lambda, \mathrm{q})=e(\lambda, \mathrm{q}),
$$

в терминах которой производящая функция интегралов движения равна

$$
F(\lambda)=-\mathcal{B}^{3} \mathcal{B}_{t t}+u(\lambda, \mathrm{q}) \mathcal{B}^{4} .
$$

Данное уравнение можно переписать в форме уравнений Ньютона

$$
\ddot{\mathcal{B}}(\lambda, \mathrm{q})=F\left(\lambda, \alpha_{1}, \ldots, \alpha_{n}\right) \mathcal{B}^{-3}(\lambda, \mathrm{q})-u(\lambda, \mathrm{q}) \mathcal{B}(\lambda, \mathrm{q}),
$$

если рассматривать интегралы движения, входящие в функцию $F(\lambda)$ как константы интегрирования. Разлагая функцию $\mathcal{B}(\lambda, q)$ в ряд по переменной $\lambda$, т.е.

$$
\mathcal{B}(\lambda, \mathrm{q})=\sum x_{j} \lambda^{j}
$$

получим, что коэффициенты $x_{j}$ этого ряда удовлетворяют ньютоновским уравнениям движения [15]. Поэтому соответствуюший гамильтониан $H$ в этих переменных имеет натуральный вид. Таким образом, декартовы переменные вводятся как коэффициенты в разложении функции $\mathcal{B}$.

В обшем случае при $v_{t} \neq 0$ функция $F(\lambda)=-\operatorname{det} L(\lambda)(4.8)$ равна

$$
F(\lambda)=\frac{1}{4 v^{2}}\left(e_{t}^{2}-2 e e_{t t}\right)+\left(\frac{v_{t}}{2 v^{2}}-w\right) \frac{e_{t} e}{v}+\left(w^{2}+\frac{u}{v}\right) e^{2}
$$

и задача об определении подходяших физических переменных остается нерешенной.

Для штеккелевских систем с матрицами Штеккеля $\mathbf{S}$, которые не могут быть получены из однородных матриц Бриля-Нетера $\mathcal{U}_{D_{s}}^{*}$, задача о построении представления Лакса в обшем виде не решена. Однако в работе [15] для систем с матрищей Штеккеля $\mathbf{S}$ (2.11) данная задача была сведена к предыдушей с помошью теории приведения или редукции абелевых интегралов. При этом соответствуюшая функция $e(\lambda)(4.4)$ приобретает неподвижные нули,

$$
e(\lambda)=\prod\left(\lambda-\lambda_{j}\right)\left(\lambda-q_{1}\right)\left(\lambda-q_{2}\right) .
$$

Здесь $\lambda_{j}$ - модули кривой $\mathcal{C}$, т.е. функции от интегралов движения.

Далее мы рассмотрим системы с $v_{t} \neq 0$, дуальные к системам, допускаюшим точечное разделение переменных с $v_{t}=0$. При этом мы ограничимся конкретными примерами, что позволит обсудить предложенное представление Лакса более детально. 


\section{5. ПРИМЕРЫ ДУАЛЬНЫХ СИСТЕМ}

В данном разделе рассматриваются штеккелевские системы на плоскости. Для систем с двумя степенями свободы мы обсудим декартовы, полярные, параболические и эллиптические системы координат [11]. В основном ограничимся изучением движения по геодезическим при $U=0(2.10)$, т.к. введение потенциала и соответствуюших функций $u(\lambda, \mathrm{q})(4.8)$ достаточно подробно обсуждалось ранее [15, 20].

5.1. Параболические и декартовы координаты $(w(\lambda, \mathrm{p}, \mathrm{q})=0)$. Рассмотрим две гиперэллиптические кривые вида

$$
\begin{aligned}
& \mathcal{C}^{(1)}: z^{2}=\prod_{i=1}^{2 g+1}\left(\lambda-\lambda_{i}\right), \\
& \mathcal{C}^{(2)}: z^{2}=\lambda^{-1} \prod_{i=1}^{2 g+1}\left(\lambda-\lambda_{i}\right) .
\end{aligned}
$$

Выбрав подходяший базис голоморфных дифференциалов, построим транспонированные симметрические матрицы Бриля-Нетера для систем с двумя степенями свободы

$$
\mathcal{U}_{1}^{* t}\left(q_{1}, q_{2}\right)=\left(\begin{array}{cc}
q_{1}^{g-1} & q_{2}^{g-1} \\
\vdots & \vdots \\
q_{1}^{2} & q_{2}^{2} \\
q_{1} & q_{2} \\
-1 & -1
\end{array}\right), \quad \mathcal{U}_{2}^{* t}\left(q_{1}, q_{2}\right)=\left(\begin{array}{cc}
q_{1}^{g-2} & q_{2}^{g-2} \\
\vdots & \vdots \\
q_{1} & q_{2} \\
1 & 1 \\
-\frac{1}{q_{1}} & -\frac{1}{q_{2}}
\end{array}\right)
$$

Различные $(2 \times 2)$-блоки матриц $\mathcal{U}_{j}^{* t}$ отвечают различным штеккелевским системам.

Для каждой из кривых (5.1) мы рассмотрим по две матрицы Штеккеля, которые отличаются друг от друга первой строкой. Выберем данные пары матриц таким образом, чтобы замена переменной времени была полностью аналогична преобразованию Колосова (1.1) [7] в смысле (2.13). Так, для кривой $\mathcal{C}^{(1)}$ выберем следуюшие матришы:

$$
\mathbf{S}_{1}=\left(\begin{array}{cc}
q_{1} & q_{2} \\
-1 & -1
\end{array}\right), \quad \widetilde{\mathbf{S}}_{1}=\left(\begin{array}{cc}
q_{1}^{2} & q_{2}^{2} \\
-1 & -1
\end{array}\right)
$$

а для второй кривой $\mathcal{C}^{(2)}$ пусть матрицы Штеккеля будут равны

$$
\mathbf{S}_{2}=\left(\begin{array}{cc}
1 & 1 \\
-\frac{1}{q_{1}} & -\frac{1}{q_{2}}
\end{array}\right), \quad \widetilde{\mathbf{S}}_{2}=\left(\begin{array}{cc}
q_{1} & q_{2} \\
-\frac{1}{q_{1}} & -\frac{1}{q_{2}}
\end{array}\right) .
$$

Введем функции Гамильтона (2.10) с $U=0$ для движения по геодезическим

$$
\begin{array}{ll}
H_{0}^{(1)}=\frac{p_{1}^{2}-p_{2}^{2}}{q_{1}-q_{2}}, & \widetilde{H}_{0}^{(1)}=\left(q_{1}+q_{2}\right)^{-1} H_{0}^{(1)}, \\
H_{0}^{(2)}=\frac{q_{1} p_{1}^{2}-q_{2} p_{2}^{2}}{q_{1}-q_{2}}, & \widetilde{H}_{0}^{(2)}=\left(q_{1}+q_{2}\right)^{-1} H_{0}^{(2)} .
\end{array}
$$


Соответствуюшие вторые интегралы движения дуальных систем связаны обшим соотношением

$$
\tilde{J}_{0}^{(k)}=J_{0}^{(k)}-\frac{q_{1} q_{2}}{q_{1}+q_{2}} H_{0}^{(k)}, \quad k=1,2 .
$$

Определим функцию $e(\lambda, \mathbf{u})(3.7)$, нули которой дают решение задачи обрашения Якоби $(2.8)$

$$
e_{1}(\lambda)=\left(\lambda-q_{1}\right)\left(\lambda-q_{2}\right), \quad e_{2}(\lambda)=\frac{\left(\lambda-q_{1}\right)\left(\lambda-q_{2}\right)}{\lambda} .
$$

Вид матриц Лакса не особенно интересен в терминах переменных разделения, поэтому сразу же перейдем к физическим переменным. Так, для кривой $\mathcal{C}^{(1)}$ используем представление (4.16)

$$
e_{1}(\lambda)=\left(\lambda-q_{1}\right)\left(\lambda-q_{2}\right)=\mathcal{B}^{2}(\lambda), \quad \mathcal{B}(\lambda)=\lambda-\frac{x}{2}-\frac{y}{4 \lambda},
$$

приводящее к следуюшей замене переменных:

$$
\begin{array}{ll}
q_{1}=\frac{x-\sqrt{2 y}}{2}, & p_{1}=p_{x}-\sqrt{2 y} p_{y}, \\
q_{2}=\frac{x+\sqrt{2 y}}{2}, & p_{2}=p_{x}+\sqrt{2 y} p_{y},
\end{array}
$$

которая очевидным образом связана с декартовыми координатами. Для второй кривой $\mathcal{C}^{(2)}$ соответствуюшее уравнение

$$
e_{2}(\lambda)=\lambda^{-1}\left(\lambda-q_{1}\right)\left(\lambda-q_{2}\right)=\lambda-x-\frac{y^{2}}{4 \lambda}
$$

определяет переход к обычным параболическим координатам

$$
\begin{array}{ll}
q_{1}=\frac{x-\sqrt{x^{2}+y^{2}}}{2}, & p_{1}=p_{x}-\frac{\sqrt{x^{2}+y^{2}}+x}{y} p_{y}, \\
q_{2}=\frac{x+\sqrt{x^{2}+y^{2}}}{2}, & p_{2}=p_{x}+\frac{\sqrt{x^{2}+y^{2}}-x}{y} p_{y} .
\end{array}
$$

В новых переменных гамильтонианы (5.5) имеют вид

$$
H_{0}^{(1)}=4 p_{x} p_{y}, \quad H_{0}^{(2)}=p_{x}^{2}+p_{y}^{2}
$$

Соответствуюшие матрицы Лакса $L_{0}(\lambda)$ построим по правилу (4.7), где функции $v\left(q_{1}, q_{2}\right)$ определим из уравнений (4.5) и (4.13):

$$
\begin{array}{lll}
v\left(\lambda, q_{1}, q_{2}\right)=1 & \text { для матриц } & \mathbf{S}_{1,2}, \\
v\left(\lambda, q_{1}, q_{2}\right)=\left(q_{1}+q_{2}\right)^{-1}=\frac{1}{x} & \text { для матриц } \widetilde{\mathbf{S}}_{1,2} .
\end{array}
$$


В физических переменных матрицы Лакса имеют вид

$$
\begin{aligned}
L_{0}^{(1)} & =\left(\begin{array}{cc}
p_{x}+(2 \lambda-x) p_{y} & \lambda^{2}-\lambda x+\frac{x^{2}-2 y}{4} \\
-4 p_{y}^{2} & -p_{x}-(2 \lambda-x) p_{y}
\end{array}\right), \\
L_{0}^{(2)} & =\left(\begin{array}{cc}
p_{x}+\frac{1}{2 \lambda} y p_{y} & \lambda-x-\frac{1}{4 \lambda} y^{2} \\
\frac{1}{\lambda} p_{y}^{2} & -p_{x}-\frac{1}{2 \lambda} y p_{y}
\end{array}\right) .
\end{aligned}
$$

Для дуальных штеккелевских систем матрицы Лакса $\tilde{L}_{0}^{(1,2)}$ равны

$$
\begin{aligned}
& \tilde{L}_{0}^{(1)}=L_{0}^{(1)}+\left(\begin{array}{cc}
0 & 0 \\
4 \frac{p_{x} p_{y}}{x} & 0
\end{array}\right)=L_{0}^{(1)}+\left(\begin{array}{cc}
0 & 0 \\
\widetilde{H}_{0}^{(1)} & 0
\end{array}\right), \\
& \tilde{L}_{0}^{(2)}=L_{0}^{(2)}+\left(\begin{array}{cc}
0 & 0 \\
\frac{p_{x}^{2}+p_{y}^{2}}{x} & 0
\end{array}\right)=L_{0}^{(2)}+\left(\begin{array}{cc}
0 & 0 \\
\widetilde{H}_{0}^{(2)} & 0
\end{array}\right) .
\end{aligned}
$$

В данном случае справедливо уравнение

$$
\left\{h_{t}(\lambda), v(\mathrm{q})\right\}=0
$$

для функции (5.7), определяющей замену времени (2.9). Вследствие этого вторая функция $w$ в определении элемента $h(\lambda)$ матрицы Лакса (4.7) равна нулю, $w=0$, и уравнение (4.9) для дуальных систем следует из соответствуюшего уравнения для систем с $v_{t}=0$ :

$$
\tilde{f}_{t}=\{\widetilde{H}, f+\widetilde{H}\}=f_{t}=0 .
$$

Заметим, что в работах [8] и [9], посвяшенных волчку Ковалевской, известные представления Лакса для дуальных систем отождествлялись с представлением Лакса для данного волчка после неканонической замены физических координат и времени. Таким образом, различные системы линеаризовались на якобиане одной и той же спектральной кривой. В отличие от этого, в нашем случае мы получаем разные представления Лакса для дуальных интегрируемых систем и соответственно разные спектральные кривые.

Можно проверить, что спектральные кривые полученных матриц Лакса $L_{0}^{(1,2)}(5.8)$ и исходные кривые $\mathcal{C}_{0}^{(1,2)}(2.4)$ при $U=0$ совпадают:

$$
z^{2}=H_{0}^{(1)} \lambda+J_{0}^{(1)}, \quad z^{2}=H_{0}^{(2)}+\frac{J_{0}^{(2)}}{\lambda} .
$$

Здесь $J_{0}^{(1,2)}$ - вторые интегралы движения, построенные по правилу $(2.2)$. Для дуальных систем с гамильтонианами $\widetilde{H}_{0}^{(1,2)}$ кривые имеют вид

$$
z^{2}=\widetilde{H}_{0}^{(1)} \lambda^{2}+\tilde{J}_{0}^{(1)}, \quad z^{2}=\widetilde{H}_{0}^{(2)} \lambda+\frac{\tilde{J}_{0}^{(2)}}{\lambda}
$$


Таким образом, для системы с гамильтонианом $H_{0}^{(1)}$ отображение Абеля однозначно на кривой (5.10). Тем не менее для той же системы на кривой вида

$$
\mathcal{C}: z^{2}=e_{2} \lambda^{2}+e_{1} \lambda+e_{0}
$$

при произвольных значениях $e_{j}$ отображение Абеля неоднозначно. Вследствие этого на данной кривой можно определить вторую дуальную систему с гамильтонианом $\widetilde{H}_{0}^{(1)}$.

Рассмотрим теперь штеккелевские системы с полиномиальным потенциалом $U$. Введем разные потенциалы для кривых (5.1) вида

$$
U^{(1)}\left(q_{j}\right)=\alpha^{2} q_{j}^{5}+\beta q_{j}^{3}, \quad U^{(2)}\left(q_{j}\right)=\alpha^{2} q_{j}^{3}+\beta q_{j}
$$

Положим $N=6$ и $N=4$ в $(4.11),(4.12)$ и рассмотрим параметрические функции вида

$$
\phi^{(1)}(\lambda)=-\alpha^{2} \lambda^{5} \quad \text { и } \quad \phi^{(2)}(\lambda)=-\alpha^{2} \lambda^{3}
$$

для кривых $\mathcal{C}^{(1)}$ и $\mathcal{C}^{(2)}$, соответственно. В обоих случаях функция $u\left(\lambda, q_{1}, q_{2}\right)$ равна $-\alpha^{2}(\lambda+2 x)$.

Теперь по правилу (4.8) мы можем построить матрицы Лакса, которые в явном виде выписывать не будем. Спектральные кривые данных матриц Лакса (4.8) совпадают с исходными кривыми (3.10). Например, соответствуюшие кривые для систем с $\widetilde{H}^{(1,2)}$ (2.4) равны

$$
\begin{aligned}
& \mathcal{C}^{(1)}: z^{2}=\alpha^{2} \lambda^{5}+\beta \lambda^{3}-\tilde{H} \lambda-\tilde{J}, \\
& \mathcal{C}^{(2)}: z^{2}=\alpha^{2} \lambda^{3}-\tilde{H} \lambda+\beta-\frac{\tilde{J}}{\lambda} .
\end{aligned}
$$

Скобки Пуассона матриц Лакса, построенных по правилам (4.7), (4.8), записьваются в обычном виде:

$$
\begin{gathered}
\{\stackrel{1}{L}(\lambda), \stackrel{2}{L}(\mu)\}=\left[r_{12}(\lambda, \mu), \stackrel{1}{L}(\lambda)\right]-\left[r_{21}(\lambda, \mu), \stackrel{2}{L}(\mu)\right], \\
r_{21}(\lambda, \mu)=-\Pi r_{21}(\lambda, \mu) \Pi
\end{gathered}
$$

Здесь П - оператор перестановки во вспомогательном пространстве $\mathbb{C}^{2} \times \mathbb{C}^{2}[21]$ и используются принятые тензорные обозначения

$$
\stackrel{1}{L}(\lambda)=L(\lambda) \otimes I, \quad \stackrel{2}{L}(\mu)=I \otimes L(\mu) .
$$

В соответствии с [15] все вычисления сводятся к вычислению всего нескольких скобок и использованию затем определений элементов матрицы Лакса (4.8).

Если отображение Абеля однозначно, то и для матриц Лакса $L_{0}^{(1,2)}(5.8)$, и для построенных по правилу $(4.8)$ матриц $L^{(1,2)}$ соответствующие $r$-матрицы $r_{i j}$ при $v_{t}=0$ состоят из двух слагаемых:

$$
r_{i j}=r_{i j}^{p}+r_{i j}^{u}
$$


Первое слагаемое - это обычная классическая $r$-матрица на алгебре петель $\mathcal{L}(s l(2))$ с рациональной зависимостью от спектрального параметра

$$
r_{12}^{p}(\lambda, \mu)=\frac{\Pi}{\lambda-\mu}=\frac{1}{\lambda-\mu}\left(\begin{array}{llll}
1 & 0 & 0 & 0 \\
0 & 0 & 1 & 0 \\
0 & 1 & 0 & 0 \\
0 & 0 & 0 & 1
\end{array}\right) .
$$

Вторая матрица $r_{i j}^{u}$ появляется для потенциальных систем, только если $u(\lambda, q) \neq 0[15]$. Появление этой второй матришы связано с автоморфизмами бесконечномерных представлений подстилающей алгебры $s l(2)$ [20], которые сдвигают спектр оператора Казимира $s l(2)$. При этом динамические $r_{i j}^{u}$-матрицы зависят только от половины динамических переменных,

$$
r_{12}^{u}=\frac{u(\lambda, \mathrm{q})-u(\mu, \mathrm{q})}{\lambda-\mu} \sigma_{-} \otimes \sigma_{-}, \quad \sigma_{-}=\left(\begin{array}{ll}
0 & 0 \\
1 & 0
\end{array}\right)
$$

Для дуальных штеккелевских систем с $v_{t} \neq 0$, т.е. и для матриц $\tilde{L}_{0}^{(1,2)}$, и для матриц $\tilde{L}^{(1,2)}$, к $r$-матрицам $(5.14)$ необходимо добавить новое слагаемое вида

$$
r_{12}^{v}=v(\lambda, \mathrm{q})\left(\begin{array}{llll}
0 & 0 & 0 & 0 \\
0 & 0 & 0 & 0 \\
0 & 1 & 0 & 0 \\
0 & 0 & 0 & 0
\end{array}\right)
$$

где матриша $r_{21}^{v}$ вычисляется по правилу (5.13). Заметим, что данная $r$-матрища пропорциональна функции $v(\lambda, \mathrm{q})$, задающей замену времени.

Слагаемое $r_{i j}^{v}$ можно связать со скрученными квантовыми $R$-матрицами Дринфельда [22]. Возьмем $R$-матрицу, которая удовлетворяет квантовому уравнению Янга-Бакстера, и построим скрученную $R$-матрицу, или твист вида

$$
\widetilde{R}=F R F_{21}^{-1}, \quad F_{21}=\Pi F \Pi,
$$

опустив зависимость от спектральных параметров. Здесь матрица $F$ удовлетворяет некоторым специальным условиям [22]. Обычным образом [23] переходя к линейной $r$-матрице, положим

$$
R=I+2 \eta r^{p}+O\left(\eta^{2}\right), \quad F=I+\eta r^{v}+O\left(\eta^{2}\right)
$$

и рассмотрим предел при $\eta \rightarrow 0$ скрученной матрицы $\widetilde{R}$

$$
\widetilde{R}_{12}=I+\eta\left(r_{12}^{p}+r_{12}^{v}-\Pi\left(r_{12}^{p}+r_{12}^{v}\right) \Pi\right)+O\left(\eta^{2}\right)
$$

С формальной точки зрения коэффициент при $\eta$ можно назвать скрученной линейной $r$-матрицей. 
В нашем случае пусть $\mathbf{h}, \mathbf{e}, \mathbf{f}$ - генераторы подстилающей алгебры $s l(2)$ :

$$
[\mathbf{h}, \mathbf{e}]=2 \mathbf{e}, \quad[\mathbf{h}, \mathbf{f}]=-2 \mathbf{f}, \quad[\mathbf{e}, \mathbf{f}]=\mathbf{h} .
$$

Введем элемент $\mathcal{F} \in U(s l(2)) \otimes U(s l(2)):$

$$
\mathcal{F}_{\xi}=\exp (\xi \cdot \mathbf{e} \otimes \mathbf{f}), \quad \xi \in \mathbb{C},
$$

из тензорного произведения соответствующих универсальных обертывающих алгебр $U(s l(2))[22]$. В фундаментальном представлении $\rho_{1 / 2}$ спина $s=1 / 2$ данный элемент равен

$$
F(\xi)=\left(\rho_{\frac{1}{2}} \otimes \rho_{\frac{1}{2}}\right) \mathcal{F}_{\xi}=\left(\begin{array}{cccc}
1 & 0 & 0 & 0 \\
0 & 1 & 0 & 0 \\
0 & \xi & 1 & 0 \\
0 & 0 & 0 & 1
\end{array}\right) .
$$

Подставим в (5.18) квантовую $R$-матрицу $X X X$-модели с рациональной зависимостью от спектрального параметра

$$
R=I+\frac{\eta}{\lambda} \Pi .
$$

Соответствуюшая скрученная матрища $\widetilde{R}$ с функцией $z(\mathrm{q})$, которая зависит от динамических переменных, использовалась в [24] для описания цепочек Тоды, ассоциированных с корневой системой $\mathcal{D}_{n}$.

Рассмотрим теперь скрученную матрицу (5.18) с функцией $\xi=v(\lambda, \mathrm{q})$, определяюшей замену времени. Мы видим, что для дуальных штеккелевских систем соответствующая линейная $r$-матрица в некотором смысле строится из скрученной линейной $r$-матрицы вида (5.19)

$$
r_{12}=r_{12}^{p}+r_{12}^{v}, \quad r_{21}=-\Pi\left(r_{12}^{p}+r_{12}^{v}\right) \Pi .
$$

Таким образом, можно сказать, что замена времени для штеккелевских систем достаточно неожиданно связана с твистом алгебры $s l(2)$ [22] и цепочками Тоды типа $\mathcal{D}_{n}[24]$.

Напомним, что в обшем случае проблема введения физических координат, в терминах которых функция Гамильтона приобретает натуральный вид, остается нерешенной, в отличие от штеккелевских систем с полиномиальными потенциалами, для которых отображение Абеля однозначно [15]. В соответствии с этим, если для матриц Штеккеля $\mathbf{S}_{1}(5.3)$ и $\mathbf{S}_{2}(5.4)$ функции Гамильтона имеют натуральный вид сразу:

$$
\begin{aligned}
& H^{(1)}=2 p_{x} p_{y}+\frac{\alpha^{2}}{4}\left(y^{2}+5 x^{2} y+\frac{5}{4} x^{4}\right)+\frac{\beta}{2}\left(\frac{3}{2} x^{2}+y\right), \\
& H^{(2)}=\frac{p_{x}^{2}}{2}+\frac{p_{y}^{2}}{2}+\frac{\alpha^{2}}{2} x\left(2 x^{2}+y^{2}\right)+\beta,
\end{aligned}
$$


то для дуальных штеккелевских систем (5.5) функции Гамильтона приобретают натуральный вид только после дополнительных преобразований координат. Так, если в первом случае достаточно преобразования одной из координат:

$$
x=\sqrt{2 \tilde{x}}, \quad p_{x}=\tilde{p}_{x} \sqrt{2 \tilde{x}},
$$

то во втором случае каноническое преобразование более сложное:

$$
\begin{array}{ll}
x=\frac{3}{2} \tilde{x}^{2 / 3}, & p_{x}=\tilde{p}_{x} \tilde{x}^{1 / 3}, \\
y=\sqrt{\frac{2}{3}} \frac{\tilde{p}_{y}}{\alpha}, & p_{y}=-\sqrt{\frac{3}{2}} \alpha \tilde{y} .
\end{array}
$$

Соответствующие функции Гамильтона $\widetilde{H}^{(1,2)}(5.5)$ равны

$$
\begin{aligned}
& \widetilde{H}^{(1)}=2 \tilde{p}_{x} p_{y}+\frac{\alpha^{2}\left(y^{2}+10 y \tilde{x}+5 \tilde{x}^{2}\right)}{8} \sqrt{\frac{2}{\tilde{x}}}+\frac{\beta(3 \tilde{x}+y)}{4} \sqrt{\frac{2}{\tilde{x}}}, \\
& \widetilde{H}^{(2)}=\frac{1}{2}\left(\tilde{p}_{x}^{2}+\tilde{p}_{y}^{2}\right)+\frac{3 \alpha^{2}}{4} \tilde{x}^{-2 / 3}\left(\frac{9}{2} \tilde{x}^{2}+\tilde{y}^{2}\right)+\beta \tilde{x}^{-2 / 3} .
\end{aligned}
$$

Система с гамильтонианом $H^{(2)}$ известна как один из интегрируемых случаев системы Энона-Эйлеса [2], а дуальная к ней система с гамильтонианом $\widetilde{H}^{(2)}$ - как система типа Холта.

Заметим, что для матрищы Штеккеля $\widetilde{\mathbf{S}}_{2}$ дополнительное каноническое преобразование координат (5.23) приводит соответствующий гамильтониан (2.10) к нормальному виду только для ограниченного семейства потенциалов $U$ (5.12). В отличие от этого для матрицы Штеккеля $\widetilde{\mathbf{S}}_{1}$ точечное преобразование (5.22) можно использовать для любых потенциалов $U$. Например, рациональному потенциалу вида

$$
U(q)=\frac{\alpha}{q^{2}}+\frac{\beta}{q}+\gamma q+\delta q^{2}+\rho q^{4}
$$

отвечает следующий гамильтониан:

$$
\widetilde{H}=2 \tilde{p}_{x} p_{y}-\frac{4 \alpha}{(\tilde{x}-y)^{2}}-\frac{\beta}{\tilde{x}-y} \sqrt{\frac{2}{\tilde{x}}}+\frac{\gamma}{4} \sqrt{\frac{2}{\tilde{x}}}+\frac{1}{2} \delta+\frac{\rho}{2}(\tilde{x}+y),
$$

к которому можно добавить слагаемые вида (5.23). Более того, данное преобразование можно применять и к неоднородным штеккелевским системам. Например, для потенциалов

$$
U_{j}\left(q_{j}\right)=\alpha_{j}+\beta_{j} q_{j}+\gamma_{j} q_{j}^{2}+\delta_{j} q_{j}^{3}, \quad j=1,2,
$$

соответствующий гамильтониан (2.10) имеет вид

$$
\begin{aligned}
\widetilde{H}= & 2 \tilde{p}_{x} p_{y}+\frac{1}{2}\left(\frac{\alpha_{2}-\alpha_{1}}{\sqrt{\tilde{x} y}}+\frac{\beta_{2}-\beta_{1}}{\sqrt{2 y}}+\frac{\beta_{2}+\beta_{1}}{\sqrt{\tilde{x}}}+\frac{\left(\gamma_{2}-\gamma_{1}\right)(\tilde{x}+y)}{2 \sqrt{\tilde{x} y}}+\gamma_{2}+\gamma_{1}\right)+ \\
& +\frac{1}{8}\left(3\left(\delta_{2}-\delta_{1}\right) \sqrt{2 y}+\left(\delta_{2}+\delta_{1}\right) \frac{\left(y^{3 / 2}+3 \tilde{x} \sqrt{y}-\tilde{x}^{3 / 2}\right)}{\sqrt{\tilde{x} y}}\right) .
\end{aligned}
$$


Неоднородные штеккелевские системы с функцией Гамильтона $\widetilde{H}(5.24)$ можно рассматривать как интегрируемые деформации кулоновской задачи или задачи Кеплера. Данные деформации близки к потенциалам типа Драша [12]. Вторые интегралы для систем типа Драша являются полиномами третьего порядка по импульсам, что отличает их от рассмотренных выше систем. Однако, т.к. при этом род соответствующей кривой (2.4) меньше числа степеней свободы, мы можем предполагать [15], что данные системы являются суперинтегрируемыми. В силу этого для гамильтониана $\widetilde{H}(5.24)$ второй интеграл движения $\tilde{J}$, по-видимому, может быть определен разными способами.

5.2. Эллиптические и полярные координаты $(w \neq 0)$. Заметим, что полярные координаты могут быть получены из эллиптических [11], и поэтому мы в основном сосредоточим внимание на эллиптических координатах. Кроме того, в полярных координатах соответствуюшая матрица Штеккеля не является симметрической.

Итак, рассмотрим гиперэллиптическую кривую вида

$$
\mathcal{C}: z^{2}=\frac{1}{(\lambda-k)(\lambda+k)} \prod_{i=1}^{2 g+1}\left(\lambda-\lambda_{i}\right), \quad k \in \mathbb{C}
$$

Выбрав подходящий базис голоморфных дифференциалов, построим транспонированные симметрические матрицы Бриля-Нетера для систем с двумя степенями свободы и выберем из них два блока вида

$$
\mathbf{S}_{3}=\left(\begin{array}{ll}
\frac{q_{1}}{q_{1}^{2}-k^{2}} & \frac{q_{2}}{q_{2}^{2}-k^{2}} \\
\frac{1}{q_{1}^{2}-k^{2}} & \frac{1}{q_{2}^{2}-k^{2}}
\end{array}\right), \quad \widetilde{\mathbf{S}}_{3}=\left(\begin{array}{cc}
\frac{4 q_{1}^{2}}{q_{1}^{2}-k^{2}} & \frac{4 q_{2}^{2}}{q_{2}^{2}-k^{2}} \\
\frac{1}{q_{1}^{2}-k^{2}} & \frac{1}{q_{2}^{2}-k^{2}}
\end{array}\right)
$$

Мы выбрали данные пары матриц таким образом, чтобы замена переменной времени была полностью аналогична преобразованию Колосова (1.1) [7].

Для полярной системы координат выберем матришы Штеккеля следуюшим образом:

$$
\mathbf{S}_{4}=\left(\begin{array}{cc}
1 & 0 \\
\frac{1}{q_{1}^{2}} & \frac{1}{4 q_{2}\left(q_{2}-1\right)}
\end{array}\right), \quad \widetilde{\mathbf{S}}_{4}=\left(\begin{array}{cc}
q_{1}^{2} & 0 \\
\frac{1}{q_{1}^{2}} & \frac{1}{4 q_{2}\left(q_{2}-1\right)}
\end{array}\right),
$$

чтобы соответствующее неканоническое преобразование времени было близко к замене времени в задаче Кеплера [11].

Движению по геодезическим при $U=0$ отвечают кривые (2.4) вида

$$
z^{2}=\frac{H_{0} \lambda+J_{0}}{\lambda-k^{2}}, \quad z^{2}=\frac{4 \widetilde{H}_{0} \lambda^{2}+\tilde{J}_{0}}{\lambda-k^{2}}
$$

где функции Гамильтона равны

$$
H_{0}^{(3)}=\frac{p_{1}^{2}\left(q_{1}^{2}-k^{2}\right)-p_{2}^{2}\left(q_{2}^{2}-k^{2}\right)}{q_{1}-q_{2}}, \quad \widetilde{H}_{0}^{(3)}=\frac{1}{4\left(q_{1}+q_{2}\right)} H^{(3)}
$$


Для полярных координат функции Гамильтона имеют вид

$$
H_{0}^{(4)}=p_{1}^{2}-4 \frac{q_{2}^{2}-k}{q_{1}^{2}} p_{2}^{2}, \quad \widetilde{H}_{0}^{(4)}=q_{1}^{-2} H_{0}^{(4)} .
$$

Введем стандартные эллиптические координаты, используя уравнение

$$
e(\lambda)=\frac{\left(\lambda-q_{1}\right)\left(\lambda-q_{2}\right)}{(\lambda-k)(\lambda+k)}=1-\frac{x^{2}}{4(\lambda-k)}-\frac{y^{2}}{4(\lambda+k)}
$$

так что

$$
\begin{aligned}
& q_{1}=\frac{x^{2}+y^{2}}{8}+\frac{1}{8} \sqrt{\left(y^{2}+x^{2}\right)^{2}+16 k\left(x^{2}-y^{2}\right)+64 k^{2}} \\
& q_{2}=\frac{x^{2}+y^{2}}{8}-\frac{1}{8} \sqrt{\left(y^{2}+x^{2}\right)^{2}+16 k\left(x^{2}-y^{2}\right)+64 k^{2}} .
\end{aligned}
$$

Соответствующее уравнение для полярных координат выглядит следующим образом:

$$
e(\lambda)=\frac{q_{1}^{2}\left(\lambda-q_{2}\right)}{\lambda^{2}-k}=-\frac{x^{2}}{4(\lambda-k)}-\frac{y^{2}}{4(\lambda+k)},
$$

откуда немедленно следует

$$
q_{1}=r=\sqrt{x^{2}+y^{2}}, \quad q_{2}=\cos ^{2}(\phi)=\frac{x^{2}}{x^{2}+y^{2}} .
$$

Как в полярных координатах, так и в эллиптических функции Гамильтона для движения по геодезическим выглядят одинаково:

$$
H_{0}=p_{x}^{2}+p_{y}^{2}, \quad \widetilde{H}_{0}=\frac{p_{x}^{2}+p_{y}^{2}}{x^{2}+y^{2}} .
$$

Далее, для построения матриц Лакса необходимо определить функции $v(\lambda, \mathrm{q})$ в (4.7) из (4.13) и (4.5):

$$
\begin{array}{ll}
v=1 & \text { для матриц } \mathbf{S}_{3,4}, \\
v=\frac{1}{4}\left(q_{1}+q_{2}\right)^{-1}=\frac{1}{x^{2}+y^{2}} & \text { для матрицы } \widetilde{\mathbf{S}}_{3}, \\
v=\frac{1}{q_{1}^{2}}=\frac{1}{x^{2}+y^{2}} & \text { для матрицы } \widetilde{\mathbf{S}}_{4} .
\end{array}
$$

Данные функции квадратичны, в отличие от функций $v$ для декартовых и параболических координатных систем (5.7).

Матришы Лакса (4.7) для обеих координатных систем можно представить в виде

$$
L_{0}(\lambda)=\left(\begin{array}{cc}
\frac{x p_{x}}{2(\lambda-k)}+\frac{y p_{y}}{2(\lambda+k)} & \epsilon \lambda-\frac{x^{2}}{4(\lambda-k)}-\frac{y^{2}}{4(\lambda+k)} \\
\frac{p_{x}^{2}}{\lambda-k}+\frac{p_{y}^{2}}{\lambda+k} & -\frac{x p_{x}}{2(\lambda-k)}-\frac{y p_{y}}{2(\lambda+k)}
\end{array}\right) .
$$


Здесь $\epsilon=0,1$ для полярных и эллиптических координатных систем, соответственно. При $\epsilon=1$ спектральная кривая данной матрицы Лакса совпадает с первой из кривых (5.27).

В отличие от декартовых и параболических координат (5.9), матрица Лакса для дуальных систем имеет более сложный вид. Данное представление строится по правилу (4.8) с ненулевой функцией $w(\mathrm{p}, \mathrm{q})$, которая выражается через функцию Гамильтона

$$
w(\mathrm{p}, \mathrm{q})=2 \sqrt{\widetilde{H}} .
$$

В явном виде для дуальных систем матрищы Лакса имеют вид

$$
\tilde{L}_{0}(\lambda)=L_{0}(\lambda)+\left(\begin{array}{cc}
w e(\lambda) & 0 \\
-2 w[h(\lambda)-w e(\lambda)-\epsilon w] & -w e(\lambda)
\end{array}\right), \quad \epsilon=0,1 .
$$

Здесь $e(\lambda)$ и $h(\lambda)$ - элементы матрицы Лакса $L_{0}(\lambda)(5.31)$. При $\epsilon=1$ спектральная кривая данной матрицы Лакса $\tilde{L}_{0}(5.33)$ совпадает со второй из кривых (5.27).

Для параболических и декартовых координат справедливо равенство

$$
\left\{\left\{H, v^{-1}(\mathrm{q})\right\}, e(\lambda, \mathrm{q})\right\}=\left\{\left\{H,\left(q_{1}+q_{2}\right)\right\}, e(\lambda, \mathrm{q})\right\}=2,
$$

которое связывает гамильтониан $H$, функцию $e(\lambda)$ и функцию $v(q)$, определяющую замену времени типа Колосова. Для полярных и эллиптических координат аналогичное уравнение имеет вид

$$
\left\{\left\{H, v^{-1}(\mathrm{q})\right\}, e(\lambda, \mathrm{q})\right\}=8(e(\lambda)-\epsilon), \quad \epsilon=0,1 .
$$

Как следствие, в данном случае функция $w(\mathrm{p}, \mathrm{q})(4.7),(5.32)$ не равна нулю. Если для декартовых и параболических координат рассмотреть более сложную замену времени, то в соответствуюшем представлении Лакса функция $w(4.7)$ также будет отличаться от нуля.

Скобки Пуассона для матрицы Лакса $L_{0}(5.31)$ замыкаются в линейную $r$-матричную алгебру (5.13) с классической $r$-матрицей (5.15) на алгебре петель $\mathcal{L}(s l(2))$ с рациональной зависимостью от спектрального параметра [21].

Скобки Пуассона между элементами дуальной матрицы $\tilde{L}_{0}(5.33)$ можно легко получить с помошью определения матрищы Лакса (4.8) в соответствии с [15]. Выпишем явно эти квадратично-линейные скобки при $\epsilon=0,1$ :

$$
\begin{aligned}
& \{e(\lambda), e(\mu)\}=0, \\
& \{h(\lambda), h(\mu)\}=\frac{2 v}{w}[e(\lambda) f(\mu)-f(\lambda) e(\mu)], \\
& \{e(\lambda), h(\mu)\}=\frac{e(\lambda)-e(\mu)}{\lambda-\mu}+\frac{4 v}{w}[h(\lambda)-w e(\lambda)] e(\mu), \\
& \{e(\lambda), f(\mu)\}=-2 \frac{h(\lambda)-h(\mu)}{\lambda-\mu}-\frac{8 v}{w}[h(\lambda)-w e(\lambda)][h(\mu)-\epsilon w], \\
& \{h(\lambda), f(\mu)\}=\frac{f(\lambda)-f(\mu)}{\lambda-\mu}-\frac{4 v}{w}[f(\lambda) h(\mu)-w f(\mu) e(\lambda)-\epsilon w f(\lambda)], \\
& \{f(\lambda), f(\mu)\}=8 v[h(\lambda) f(\mu)-h(\mu) f(\lambda)]-\epsilon w[f(\lambda)-f(\mu)] .
\end{aligned}
$$


Данные скобки можно представить в виде квадратично-линейной $r$-матричной алгебры вида

$$
\begin{aligned}
&\{\stackrel{1}{L}(\lambda), \stackrel{2}{L}(\mu)\}=\left[r_{12}, \stackrel{1}{L}(\lambda)\right]+\left[r_{21}, \stackrel{2}{L}(\mu)\right]+ \\
&+R \stackrel{1}{L}(\lambda) \stackrel{2}{L}(\mu)+\stackrel{1}{L}(\lambda) \stackrel{2}{L}(\mu) R-\stackrel{1}{L}(\lambda) R \stackrel{2}{L}(\mu)-\stackrel{2}{L}(\mu) R \stackrel{1}{L}(\lambda) .
\end{aligned}
$$

Здесь линейная $r$-матрица

$$
r_{12}(\lambda, \mu)=r_{12}^{p}(\lambda-\mu)+4 \epsilon v r_{12}^{w}, \quad r_{21}(\lambda, \mu)=-\Pi r_{21}(\lambda, \mu) \Pi
$$

состоит из обычной $r$-матрицы $r^{p}(\lambda-\mu)$ на алгебре петель $\mathcal{L}(s l(2))$ и дополнительного динамического слагаемого $r^{w}$, пропорционального $\epsilon=0,1$,

$$
r_{12}^{w}=\left(\begin{array}{cccc}
0 & 0 & 0 & 0 \\
0 & 0 & 0 & 0 \\
w & 1 & 0 & 0 \\
0 & -w & 0 & 0
\end{array}\right) .
$$

Вторая матрица $R$, входящая в квадратичные слагаемые, имеет вид скрученной динамической матрицы (5.19)

$$
R=-\frac{2 v}{w}\left(r_{12}^{w}+r_{21}^{w}\right)=-\frac{2 v}{w}\left(r_{12}^{w}-\Pi r_{12}^{w} \Pi\right) .
$$

Более подробно рассмотреть соответствуюшие $r$-матричные алгебры в классическом и квантовом случаях мы планируем в дальнейшем.

Для систем с потенциалом $U \neq 0$ функция $u(\lambda, \mathrm{q})$ строится обычньмм образом $[15,20]$ и поэтому представления Лакса вида (4.8) здесь вьписываться явно не будут. Как обычно, для дуальных штеккелевских систем (5.29) функции Гамильтона приобретают натуральный вид только после дополнительного преобразования координат:

$$
\begin{array}{llrl}
x & =\sqrt{\tilde{x}}-\sqrt{\tilde{y}}, & p_{x}=\sqrt{\tilde{x}} \tilde{p}_{x}-\sqrt{\tilde{y}} \tilde{p}_{y}, \\
y=-i(\sqrt{\tilde{x}}+\sqrt{\tilde{y}}), & p_{y}=i\left(\sqrt{\tilde{x}} \tilde{p}_{x}+\sqrt{\tilde{y}} \tilde{p}_{y}\right) .
\end{array}
$$

Так, однородный квадратичный потенциал

$$
U^{(3)}\left(q_{j}\right)=\alpha q_{j}^{2}+\beta q_{j}+\gamma
$$

порождает следующий дуальный гамильтониан для эллиптических координат:

$$
\widetilde{H}^{(3)}=\tilde{p}_{x} \tilde{p}_{y}+\frac{\alpha}{4}(\tilde{x}+k)(\tilde{y}+k)-\frac{\beta}{8 \sqrt{\tilde{x} \tilde{y}}}(2 \tilde{x} \tilde{y}+k \tilde{x}+k \tilde{y})+\gamma,
$$

аналог которого для полярных координат имеет вид

$$
\begin{aligned}
\widetilde{H}^{(4)}= & \tilde{p}_{x} \tilde{p}_{y}+\frac{\alpha}{64} \frac{\left(\tilde{x}^{2}-\tilde{y}^{2}\right)^{2}}{\tilde{x}^{3} \tilde{y}^{3}}-\frac{\gamma k^{2}}{16}\left(\frac{4}{k^{2} \sqrt{\tilde{x} \tilde{y}}}+\frac{1}{\tilde{x}^{2}}+\frac{1}{\tilde{y}^{2}}-\frac{2}{\tilde{x} \tilde{y}}\right)+ \\
& +\frac{\beta k^{3}}{32}\left(\sqrt{\frac{\tilde{x}}{\tilde{y}^{5}}}+\sqrt{\frac{\tilde{y}}{\tilde{x}}}-\frac{1}{\sqrt{\tilde{x} \tilde{y}^{3}}}-\frac{1}{\sqrt{\tilde{y} \tilde{x}^{3}}}-\frac{16 i}{k^{3} \tilde{x}^{1 / 4} \tilde{y}^{1 / 4}}\right) .
\end{aligned}
$$

Данные системы можно рассматривать как интегрируемые деформации задачи Кеплера. 


\section{6. ЗАКЛЮЧЕНИЕ}

В настоящей работе изучаются неканонические преобразования переменной времени, которые связывают различные интегрируемые штеккелевские системы. Для некоторых из систем предложена конструкция $(2 \times 2)$-представления Лакса и построены соответствующие $r$-матричные алгебры. Данную конструкцию можно рассматривать как деформацию стандартного алгебро-геометрического метода построения матриц Лакса в виде элементов пополненной алгебры петель $\mathcal{L}(s l(2))$. Соответствующие $r$-матричные алгебры являются квадратично-линейными, а входящие в них $r$-матрицы зависят от динамических переменных.

Следует заметить, что предложенные замены времени в виде отношения определителей матриц Штеккеля (2.9) не исчерпывают всех возможных неканонических преобразований, которые сохраняют интегрируемость. В качестве примера можно рассмотреть само преобразование Колосова для волчка Ковалевской [7]. Напомним, что данное преобразование связывает штеккелевскую систему с другой интегрируемой системой, которую невозможно привести к штеккелевскому виду. Таким образом, встает вопрос об описании различных интегрируемых систем, связанных со штеккелевскими системами неканоническими преобразованиями общего вида.

Одним из важных моментов, на наш взгляд, является использование различных представлений Лакса для взаимосвязанных различных штеккелевских систем. Напомним, что данные матрицы Лакса в явном виде включают в себя функцию $v(q)$, отвечающую за замену времени. Мы надеемся, что такой подход может быть использован не только для штеккелевских интегрируемых систем. Поэтому в дальнейшем автор намерен рассмотреть представления Лакса для произвольных интегрируемых систем, связанных со штеккелевскими системами заменой времени общего вида.

Благодарности. Автору приятно поблагодарить И. В. Комарова за многочисленные полезные обсуждения и интерес к работе. Работа выполнена при финансовой поддержке Министерства образования РФ, грант № 97-40-1.14-25.

\section{Список литературы}

[1] С. В. Ковалевская. Научные работы. М.: Изд-во АН СССР, 1948.

[2] A. Ramani, B. Grammaticos, T. Bountis. Phys. Rep. 1989. V. 180. № 3. P. 159.

[3] E. L. Ince. Ordinary Differential Equations. New York: Dover, 1956.

[4] О.И. Богоявленский. Методы качественной теории динамических систем в астрофизике и газовой динамике. М.: Наука, 1980.

[5] M. Adler, P. van Moerbeke. Invent. Math. 1989. V. 97. P. 3.

[6] С. И. Зиглин. Функц. анализ и его прилож. 1982. Т. 16. № 3. С. 30; 1983. Т. 17. № 1. С. 1.

[7] Г. В. Колосов. Тр. Отд. физ. наук Общества любителей естествознания. 1901. Т. 11(1). С. 5.

[8] M. Adler, P. van Moerbeke. Commun. Math. Phys. 1988. V. 113. P. 659.

[9] L. Haine, E. Horozov. Physica D. 1987. V. 29. P. 173.

[10] J. Moser. Commun. Pure Appl. Math. 1970. V. 23. P. 609.

[11] Г. Голдстейн. Классическая механика. М.: Гостехиздат, 1957.

[12] J. Drach. C. R. Acad. Sci. Paris. 1935. V. 200. P. 22.

[13] P. Stäckel. C. R. Acad. Sci. Paris. 1893. V. 116. P. 485, 1284; V. 121. P. 489. 
[14] В. И. Арнольд. Математические методы классической механики. Изд. 3-е. М.: Наука, 1989.

[15] А. В. Цыъанов. ТМф. 1998. Т. 115. № 1. С. 3.

[16] Б. А. Дубровин. УМН. 1981. Т. 36. С. 11.

[17] V. M. Buchstaber, V.Z. Enolskii, D. V. Leykin. Rev. Math. and Math. Phys. 1997. V. 10. P. 1.

[18] M. Noether. Math. Ann. 1887. V. 28. P. 354.

[19] O. Bolza. Am. J. Math. 1895. V. 17. P. 11.

[20] A.V. Tsiganov. J. Math. Phys. 1998. V. 39. P. 650.

[21] Л. А. Тахтаджян, Л. Д. Фаддеев. Гамильтонов подход в теории солитонов. М.: Наука, 1986.

[22] S. Khoroshkin, A. Stolin, V. Tolstoy. Rational solutions of Yang-Baxter equation and deformation of Yangians. In: From Field Theory to Quantum Groups. Eds. B. Jancewicz and J. Sobczyk. River Edge, N. Y.: World Scientific, 1996. P. 53.

[23] A. V. Tsiganov. J. Phys. A. 1994. V. 27. P. 6759.

[24] A. V. Tsiganov. J. Phys. A. 1998. V. 31. P. 8049. 